\title{
Assessing stability in bioanalysis: reflections on the last 10 years
}

\author{
Chad Briscoe*,1 \\ ${ }^{1}$ Bioanalytical Sciences, PRA Health Sciences, 11070 Strangline Road, Lenexa, KS 66215, USA \\ *Author for correspondence: briscoechad@prahs.com



First draft submitted: 18 February 2019; Accepted for publication: 26 February 2019; Published online: 4 April 2019

Keywords: FDA • regulation • stability • validation

10 years ago, I was asked to write a review on the factors affecting the stability of drugs and metabolites for a new journal, Bioanalysis. At that time, I thought what an interesting and at the same time boring topic. However, it is very critical to what we do in the field of bioanalysis so I agreed to write it knowing that as the worst case, I will learn something myself, which in fact I certainly did. When writing an exhaustive review, you are inclined to read dozens if not hundreds of abstracts and papers. Of course, it was also a very nice opportunity to contribute to the new journal, with a singular focus on my field of science. It is gratifying to see that as of writing this follow-up editorial, the original article [1] has been downloaded 571-times and cited in 39 subsequent articles based on a search in google scholar. It seems that there has been significant interest in the topic over the years.

What I would never have predicted is how much the topic of stability would change in the subsequent 10 years. This is a topic that one would expect to stay static or should I say, stable. Papers relating to specific challenges with an assay would be expected but the physical properties affecting stability do not change of course. What has changed dramatically in the subsequent 10 years is the types of molecules we study, how we study them and the regulations, which dictate how we do the stability testing. In fact, these topics have changed so much that we are planning an upcoming issue of Bioanalysis edited by Rafiq Islam and myself, which will focus completely on this topic.

The review article which I wrote in the first issue of Bioanalysis in 2009 was focused almost exclusively on stability issues related to small molecules with just a short section mentioning biologics. As the landscape of drug development has shifted toward more biologics of different constructs, we have seen new challenges emerging. In fact, we find that a large portion of the upcoming focus issue is devoted to issues related to large molecules.

I hope you will find this editorial thought provoking and a useful preview for the upcoming themed issue on stability. I found it interesting to consider how our work has changed in this field in those areas mentioned previously: types of molecules, how we study the molecules and how the regulations have evolved. The study of large molecules has brought issues with how to conduct stability with specific types of molecules/therapies and how to conduct stability tests differently with some of the new technologies that have emerged. Although not an exhaustive list, these new types of therapies include antibody-drug conjugates (ADCs), stability of antidrug antibodies (ADAs), oligonucleotides and cellular therapies. While LC-MS/MS and ligand-binding assays are used for the analysis of several of these, other technologies such as flow cytometry and qPCR are now also coming into the focus of bioanalytical scientists.

The first ADC was approved in 2001 but future approvals did not come until 2011 and later with only four approved to date. Therefore, it is only recently that ADCs have been on the radar of a large number of bioanalytical chemists with an estimated 175 currently in development [2]. ADCs come with a few unique challenges in terms of stability. First of all, stability must be measured for the antibody, the intact ADC and the payload. When measuring the stability of these species, one must consider the source of any instability detected. For instance, instability in the ADC may be due to a deconjugation of the linker or an instability of the antibody or payload itself. It could 
also be that processing of the samples leads to cleavage of the linker. When measuring stability of the payload, it should also be considered that competing mechanisms of deconjugation leading to an increase in circulating payload may mask actual chemical instability of the molecule itself. Carefully constructed experimentation and sample processing procedures should lead to reliable results.

In 2009, it was not generally considered necessary to conduct stability experiments on ADAs. This is because immune reactions generating ADAs are polyclonal. Consequently, it is not practically possible to have authentic reference standards for comparison in a stability experiment. Additionally, antibodies are generally considered to be highly stable, so when considering these two factors together, it was considered that stability testing was unnecessary. However, the exponential growth in biologics drug development and therefore immunogenicity testing led the US FDA to issue a guidance on immunogenicity testing in 2016. This draft guidance called for assessment of stability, which was carried into the final guidance, which was released in January 2019 [3]. The guidance statesthat "studies evaluating short-term stability, including, as relevant, freeze-thaw cycle and refrigerator-and room-temperature stability of positive control antibodies, may be useful." It is commonly accepted that suggestions made in FDA guidance documents are generally followed as requirements and, thus, stability testing for ADAs has become common in most laboratories conducting regulated immunogenicity testing.

Oligonucleotides have been in development for over 30 years with the first approval, Spinraza ${ }^{\circledR}$, only coming in 2016 [4]. Over the course of that time, more and more oligonucleotide therapeutics have been in development. Oligonucleotides are commonly measured in bioanalytical assays using either a hybridization ELISA approach or LC-MS/MS. Additionally, because nucleases are present that degrade DNA, most are modified for stability in the delivery mechanism [5]. Due to these factors, it is important that additional consideration be given to the assessment of stability in oligonucleotide therapeutics.

Cellular therapy is another rapidly emerging drug technology that merits special consideration. Cellular therapies typically utilize qPCR and flow cytometry assays. This is a completely novel therapeutic approach, which utilizes two techniques that are not new but relatively new to most bioanalytical laboratories and require significant consideration and discussion of how to approach stability testing.

Biomarkers are another area that merits discussion. As biomarkers have gained increasing attention in clinical trials, the FDA has taken notice. The 2018 FDA guidance [6] now includes a section on biomarkers. For pivotal biomarkers, this means that the same stability assessments must be completed as for PK assessments. This can be a challenge in the biomarker space where endogenous measurements, lack of authentic reference standards, use of commercial kit applications and selectivity of assays can provide additional challenges. Also, as mentioned previously, new technologies such as flow cytometry, qPCR and many others would seemingly be applicable under such rules. How the many types of laboratories will comply with this requirement in many different techniques is yet to be fully worked out.

Flow cytometry and qPCR while completely different technologies, are similar in that they are emerging as important techniques in clinical development for measurement of primary and secondary end point biomarkers. In the case of cellular therapy, they are measuring the proliferation of the genes and the treated cell populations and therefore are in a sense measuring the PKs. As such, these technologies, which are not mentioned whatsoever in the various guidance documents for regulated bioanalysis, are critical end points in clinical trials. Full consideration of the validation of the assays including, of course, stability assessments must be conducted.

It is also interesting to look back over the last 10 years and consider how the regulatory expectations around stability testing have evolved beyond just considerations for biomarkers. In some cases, this is an interpretation of past guidance documents, such as thoroughly assessing "the stability of the analytes during sample collection," which was part of the 2001 FDA guidance [7]. Despite this being a part of the FDA guidance for many years, in the course of my personal observations, the assessment of stability in whole blood was not adopted as standard practice until late in the 2000's. There were likely various reasons for this. On the practical side, there were thoughts that it was only the responsibility of the bioanalytical laboratory to ensure sample validity from the time they receive a sample. Also, there were concerns that to do a truly valid assessment, one would have to have a whole blood assay developed and validated. Without this would the data even be deemed acceptable? Testing stability in the collection and plasma separation process can be complex. There are questions regarding whether the stability issues that might be detected are due to instability or partitioning of compound into blood cells. For example, in my own laboratory we have seen occurrences of stability tests failing in 'on ice' conditions while passing the warmer 'room temperature' conditions, which intuitively indicates that it is not a stability problem. In recent years, the assessment of stability has become common, but the approach is still not fully agreed upon and problems are still encountered [8-11]. 
The current expectation, and I believe industry standard approach, is that during assay validation, stability evaluations should cover the expected sample conditions before receipt at the analytical site (e.g., at the clinical site, during shipment and at all other secondary sites) as well as during receipt and analysis at the analytical site [6]. As stability of sample collection has gained increasing regulatory scrutiny, regulators have recently assigned additional scrutiny on the entire lifespan of a sample. In 2019, the expectation is that bioanalytical laboratories will take responsibility for the stability of the sample from collection through processing, shipping, receipt at the bioanalytical laboratory, instrumental analysis and long-term storage.

Another issue that has emerged in recent years is the Health Canada requirement to conduct long-term storage stability in triplicate tubes. This came about in a 2015 declaration from Health Canada [12,13]. The premise of the Canadian Letter was that when conducting long-term frozen storage stability multiple aliquots must be assessed. The requirement comes from the assumption that measurement from a single frozen tube does not properly account for variability which may occur during storage. Following several years of discussion and several publications (many which argued against the necessity of such a rule) I believe that this too has become standard practice in most regulated bioanalytical laboratories for applicable study types.

Other areas of stability assessment in assay validation have also gained increased attention including stability in co-administered compounds, stock solutions and reference standards where the standard practice has grown more extensive.

I wonder if in another 10 years I will be writing another update like this one. What has changed in regulations? What new drug constructs have been developed? How have the emerging topics like cellular therapies and biomarkers been treated by the global regulatory agencies? Looking back at these past 10 years, what I am certain of is that there will be changes. It is clear to me that the field of stability testing is not, in fact, a stable one.

\section{Financial \& competing interests disclosure}

The author has no relevant affiliations or financial involvement with any organization or entity with a financial interest in or financial conflict with the subject matter or materials discussed in the manuscript. This includes employment, consultancies, honoraria, stock ownership or options, expert testimony, grants or patents received or pending, or royalties.

No writing assistance was utilized in the production of this manuscript.

\section{References}

1. Briscoe CJ, Hage DS. Factors affecting the stability of drugs and drug metabolites in biological matrices. Bioanalysis 1(1), 205-220 (2009).

2. The expanding field of antibody-drug conjugate. https://adcreview.com/news/expanding-field-antibody-drug-conjugates/

3. US Department of Health and Human Services, Food and Drug Administration, Center for Drug Evaluation and Research (CDER). Immunogenicity testing of therapeutic protein products - developing and validating assays for anti-drug antibody detection guidance for industry

(2019). https://www.fda.gov/ucm/groups/fdagov-public/@fdagov-drugs-gen/documents/document/ucm629728.pdf

4. Thirty years of innovation pays off as oligonucleotide therapeutics come to market. https://medicalxpress.com/news/2018-01-years-oligonucleotide-therapeutics.html

5. Xu L, Anchordoquy T. Drug delivery trends in clinical trials and translational medicine: challenges and opportunities in the delivery of nucleic acid-based therapeutics. J. Pharm. Sci. 100(1), 38-52 (2011).

6. US Department of Health and Human Services, Food and Drug Administration, Center for Drug Evaluation and Research (CDER). Bioanalytical method validation guidance for industry (2018). https://www.fda.gov/downloads/drugs/guidances/ucm070107.pdf

7. US Department of Health and Human Services, Food and Drug Administration Center for Drug Evaluation and Research (CDER) Center for Veterinary Medicine (CVM). Guidance for industry bioanalytical method validation (2001).

8. Freisleben A, Brudny Klöppel M, Mulder H, de Vries R, de Zwart M, Timmerman P. Blood stability testing: European Bioanalysis Forum view on current challenges for regulated bioanalysis. Bioanalysis 3(12), 1333-1336 (2011).

9. Ginny J, Elizabeth P, Curtis ES, Chris K, Elliot O. Unexpected results for sample collection and handling stability assessment for sumatriptan in human plasma. Presented at WRIB, Montreal, QC, Canada, April, 2013. https://www.celerion.com/wp-content/uploads/2013/04/Celerion_WRIB_040913_Unexpected-Results-for-Sample-Collection.pdf

10. Redrup MJ, Igarashi H, Schaefgen J et al. Sample management: recommendation for best practices and harmonization from the global Bioanalysis Consortium Harmonization Team. AAPS J. 18(2), 290-293 (2016).

11. Hilhorst M, van Amsterdam P, Heinig K, Zwanziger E, Abbott R. Stabilization of clinical samples collected for quantitative bioanalysis a reflection from the European Bioanalysis Forum. Bioanalysis 7(3), 333-343 (2015). 
12. Notice: Clarification of Bioanalytical Method Validation Procedures. Health Canada, Bureau of Pharmaceutical Sciences Therapeutic Products Directorate, ON, Canada (2015). https://www.canada.ca/en/health-canada/services/drugs-health-products/drug-products/an nouncements/notice-clarification-bioanalytical-method-validation-procedures.html

13. Addendum to Notice: Clarification of Bioanalytical Method Validation Procedures. Health Canada, Bureau of Pharmaceutical Sciences Therapeutic Products Directorate, ON, Canada (2016). www.hc-sc.gc.ca/dhp-mps/prodpharma/activit/announce 